\title{
Correlation between adiponectin level and the degree of fibrosis in patients with non-alcoholic fatty liver disease
}

\author{
Manal Sabry Mohamed ${ }^{1}$, Tarek Mohammed Youssef ${ }^{1}$, Esraa Ebrahim Abdullah ${ }^{1}$ and \\ Ahmed Elmetwally Ahmed ${ }^{1,2^{*}}$ (D)
}

\begin{abstract}
Background: Non-alcoholic fatty liver disease (NAFLD) is one of the most prevalent chronic liver diseases, particularly in Egypt. It is defined as the accumulation of lipids inside the hepatocytes, in the absence of other etiologies of hepatic damage. It is frequently associated with obesity, diabetes mellitus, and metabolic syndrome. Adiponectin is an abundant adipocyte-derived protein with well-established anti-atherogenic, insulin-sensitizing, and anti-inflammatory properties. The liver is a major target organ for adiponectin especially in fatty liver diseases, and this adipocytokine has the ability to control many liver functions including metabolism, inflammation, and fibrosis. In this study, we aimed to find out the correlation between the degree of liver fibrosis in NAFLD patients and their serum adiponectin level as a future non-invasive method for the assessment of liver fibrosis to substitute liver biopsy to avoid its hazardous complication and also to study the correlation between diabetes mellitus as well as obesity and serum adiponectin level.
\end{abstract}

Results: Fifty patients were selected to participate in our study based on our inclusion criteria. They were recruited from the Internal Medicine Department, Gastroenterology Clinic in Al-Demerdash Hospital using a convenient sampling method. Diagnosis of NAFLD was confirmed by laboratory markers: aspartate aminotransferase (AST), alanine aminotransferase (ALT), lipid profile, ultrasound, and FibroScan examination. Analyzing the adiponectin levels showed that besides its significant correlation with body mass index (BMI), hypertension, diabetes mellitus, and dyslipidemia, it was significantly lower in the high-grade fibrosis group compared to the low-grade fibrosis group with a $P$-value of (0.000) and a cutoff value for stage $3 / 4$ fibrosis of about $2.31 \mu \mathrm{g} / \mathrm{ml}$ which marked a promising hope of adiponectin being of protective value against liver fibrosis.

Conclusion: Both serum levels and hepatic adiponectin receptor expression are decreased in NAFLD. Therefore, either adiponectin itself or adiponectin-inducing agents might be of key therapeutic interest in the near future in the treatment of NAFLD.

Keywords: Non-alcoholic fatty liver disease, Hepatic stellate cells, Adenosine monophosphate-activated protein kinase

\footnotetext{
*Correspondence: adhammetwally@hotmail.com

${ }^{1}$ Gastroenterology, Hepatology and Internal Medicine Department, Ain

Shams University, Cairo, Egypt

Full list of author information is available at the end of the article
}

\begin{abstract}
Background
Non-alcoholic fatty liver disease (NAFLD) represents a spectrum of disorders characterized by macrovescicular hepatic steatosis occurring in individuals without relevant alcohol consumption. It is a complex metabolic condition in which both lifestyle and genetic factors have a pathogenic role and has been increasingly
\end{abstract}


recognized as a major cause of liver-related morbidity and mortality [29].

Moreover, NAFLD has been convincingly associated with the metabolic insulin resistance syndrome; most patients are overweight or frankly obese, with altered glucose regulation, dyslipidemia, and raised blood pressure, all contributing to the disorder [22].

Insulin resistance, through the inhibition of lipid oxidation and increased fatty acid and triglycerides synthesis, is believed to be a key factor in the development of fatty liver. Moreover, insulin resistance states, such as obesity and diabetes, are also characterized by elevated expression and production of several cytokines; of particular interest are cytokines produced by adipose tissue, the so-called adipokines (adiponectin, leptin, resistin), nuclear factor $\alpha(\mathrm{NF} \alpha)$, transforming growth factor $\beta$ (TGF $\beta$ ), and plasminogen activator inhibitor-1 (PAI-1). Their role in the development of diabetes and other obesity complication has been suggested [35].

Adiponectin, a $28-\mathrm{kDa}$ protein adipocytokine, is mainly produced and secreted into the circulation by white adipose tissue. The primary function of adiponectin is the regulation of carbohydrate and lipid metabolism. However, the full extent of its biological action remains to be elucidated, with a variety of effects on different cell and tissue types, including its immunemodulatory, anti-inflammatory, and anti-fibrotic properties [32].

Regarding its protective effects, adiponectin regulates hepatic stellate cell (HSC) proliferation, as well as migration, and induces their apoptosis through the activation of adenosine monophosphate-activated protein kinase (AMPK). Moreover, adiponectin can attenuate HSC activation and suppress the expression of pro-fibrogenic genes, including collagen I, TGF- $\beta 1$, and alpha-smooth muscle actin ( $\alpha$-SMA), leading to the inhibition of liver fibrogenesis. With such potent effects on HSCs against liver fibrosis, adiponectin may be developed as a novel therapeutic agent in liver fibrosis. As it can be detected in the circulation and exerts its effects on various cells, it may have prognostic and diagnostic values for several human diseases. Interestingly, hypo-adiponectinemia has been documented as highly prevalent in patients with NAFLD and liver fibrosis, thereby establishing the possible influence of adiponectin levels in the development and progression of liver fibrosis [34].

Nevertheless, the underlying mechanisms of the association between adiponectin and liver fibrosis have yet to be completely elucidated. Therefore, the purpose of this study is to find the correlation between adiponectin and the degree of liver fibrosis in patients with NAFLD as a future substitution for liver biopsy to avoid its hazardous complications and also to study the correlation between diabetes mellitus as well as obesity and serum adiponectin level.

\section{Methods}

This was a cross-sectional study conducted on 50 Egyptian patients with a diagnosis of NAFLD recruited from Ain Shams University, Internal Medicine Department, Gastroenterology Clinic, between January 2019 and February 2020 using a convenient sampling method. An informed written consent was obtained from all patients before inclusion in this study. The study was approved by the Ethical Review Board of our institution. The study protocol conforms to the ethical guidelines of the Declaration of Helsinki as reflected in a priori approval by the institution's human research committee.

Diagnosis of NAFLD was confirmed by the evidence of hepatic steatosis on imaging, abnormal liver enzymes (ALT > $52 \mathrm{IU} / \mathrm{l}, \mathrm{AST}>39 \mathrm{IU} / \mathrm{l})$, and elevated lipid profile in the absence of secondary causes of hepatic fat accumulation such as significant alcohol consumption or longterm use of steatogenic medications.

\section{Exclusion criteria}

The exclusion criteria were patients with other diagnosed chronic liver diseases: chronic viral hepatitis, metabolic and genetic disorders as hemochromatosis and Wilson disease, autoimmune hepatitis, drug-induced liver disease, alcoholic liver disease due to significant alcohol consumption, and celiac disease.

All patients were subjected to the following: thorough history taking and full clinical examination. Investigations included complete blood count $(\mathrm{CBC})$, alanine aminotransferase (ALT), aspartate aminotransferase (AST), serum total and direct bilirubin, serum albumin, international normalization ratio (INR), hepatitis B surface antigen ( $\mathrm{HbsAg}$ ), hepatitis $\mathrm{C}$ virus antibody ( $\mathrm{HCV}$ $\mathrm{Ab})$, fasting blood sugar, and glycosylated hemoglobin (HbA1C), in addition to lipid profile which included triglyceride, total cholesterol, high-density lipoprotein (HDL), and low-density lipoprotein (LDL). Abdominal ultrasound was done for the diagnosis of steatosis and steatohepatitis. The criteria for the diagnosis of steatohepatitis include presence of $>5 \%$ macrovesicular steatosis, inflammation, and liver cell ballooning. Assessment of liver fibrosis was done by transient elastography (FibroScan) (Table 1).

In the assessment of plasma adiponectin level using the Human Adiponectin ELISA Kit (Chongqing Biospes Com, Ltd.), the following was performed after collecting blood samples from our participants: Plasma samples were isolated from patients soon after the collection then stored at $-20^{\circ}$ for further processing. EDTA was used as the anticoagulant. The sample was 
then diluted using the provided diluent buffer. A concentrated wash buffer was diluted using distilled water. A standard solution for lyophilized human adiponectin was prepared by adding the sample to the standard diluent buffer. The biotin-conjugated anti-human adiponectin antibody was prepared by dilution with antibody diluent buffer. The avidin-biotin-peroxidase was prepared by dilution with $\mathrm{ABC}$ diluent buffer. All the previously prepared kit components are added to the wells at the provided order and timings mentioned in the kit manual. Then, the readings are calculated with a specific equation, and curves are plotted using standard readings and readings of the samples at different wells. The human adiponecin concentration of the samples was interpolated from the standard curve.

\section{Data collection and analysis}

All the previous data were collected in a spreadsheet using Microsoft Excel 2013 for each individual patient including age, sex, height, weight, BMI, SBP, DBP, $\mathrm{CBC}$, fasting and 2-h post-prandial (2hPP) blood sugar, HbA1C, AST, ALT, total and direct bilirubin, serum albumin, INR, lipid profile, serum adiponectin level, and FibroScan results.

Patients were categorized according to the previous data into diabetic/non-diabetic, overweight/obese, and low-grade/high-grade fibrosis.

For each of these groups, correlations were made between the dependent variable (diabetes, BMI, grade of fibrosis) and all other obtained clinical data. Serum adiponectin level was reported in all patients and correlated as a dependent variable with all other clinical data including FibroScan results. For data analysis, the SPSS software was used. All numerical data were represented as means and standard deviations. All categorical data were represented as percentages. Regression analysis was performed to assess the correlation between different variables where $P$-value $<0.05$ indicated significant correlation. Diagnostic accuracy tests were also performed to assess the use of adiponectin as an indicator for fibrosis, diabetes, and obesity. Sensitivity, specificity, positive predictive value (PPV), negative predictive value (NPV), and receiver operating characteristic (ROC) analysis were performed for that matter.

Table 1 Grading of fibrosis in NAFLD patients according to FibroScan measurements

\begin{tabular}{lllll}
\hline & F0-F1 & F2 & F3 & F4 \\
\hline NAFLD & $2-7 \mathrm{kPa}$ & $7.5-10 \mathrm{kPa}$ & $10-14 \mathrm{kPa}$ & $14 \mathrm{kPa}$ or higher \\
\hline
\end{tabular}

\section{Results}

This study included 50 patients with a diagnosis of NAFLD recruited from Ain Shams University, Internal Medicine Department, Gastroenterology Clinic. Among the 50 patients, 28 patients were overweight (BMI over $25 \mathrm{~kg} / \mathrm{m}^{2}$ ) and 22 patients were obese (BMI over $30 \mathrm{~kg} /$ $\mathrm{m}^{2}$ ) The patients' demographic characteristics, anthropometric measures, and laboratory investigations are summarized in Table 2.

All those previously listed demographic, clinical data, and laboratory investigations were further correlated to the level of serum adiponectin and degree of liver fibrosis measured by FfibroScan.

Table 3 shows the mean adiponectin level was $4.08 \pm 1.32 \mu \mathrm{g} / \mathrm{ml}$ in the studied group. Ten patients (20\%) showed high-grade fibrosis using FibroScan score (F3 and F4), while the majority of the patients (80\%) showed low-grade fibrosis (F0, F1, and F2).

A significant correlation was found between obesity and DM with a $P$-value of 0.014 . No significant correlation was found between obesity and HTN according to the collected data (Tables 4 and 5).

A highly significant correlation was found between BMI and diabetes mellitus (DM) as expected where higher BMI values were reported among diabetic patients compared to the non-diabetic group $(30.56 \pm 2.6$ vs $28.6 \pm 1.81$, respectively) with a $P$-value of 0.005 as shown in Table 6.

The exact distribution of grades F0-F4 is demonstrated in Table 7 which shows that all non-diabetic patients had low-grade fibrosis (F0, F1, and F2), while about 34.5\% of diabetic patients scored high-grade fibrosis (F3-F4) establishing a highly significant positive correlation between diabetes and high-grade fibrosis with a $P$-value of 0.000 .

Table 8 shows a highly significant correlation between the grade of liver fibrosis and BMI and diabetes. A significant correlation was found between hypertension and the grade of fibrosis by FibroScan.

Table 9 shows a highly significant positive correlation between adiponectin level and platelet, albumin, and high-density lipoprotein (HDL); a highly significant negative correlation with BMI, HbA1C, total cholesterol, triglyceride (TG), and low-density lipoprotein (LDL); a significant negative correlation with SBP; and a non-significant negative correlation with DBP.

Table 10 shows the exact distribution of adiponectin levels among patients with different grades of fibrosis demonstrating a statistically significant negative correlation between adiponectin level and grade of liver fibrosis measured by FibroScan.

A cutoff value of 2.31 was determinant between lowgrade and high-grade fibrosis, meaning that all our 
Table 2 Demographic characteristics, anthropometric measures, and laboratory investigations of the studied cases

\begin{tabular}{|c|c|c|}
\hline & & Total no. $=50$ \\
\hline \multirow[t]{2}{*}{ Sex } & Females & 17 (34.0\%) \\
\hline & Males & $33(66.0 \%)$ \\
\hline \multirow[t]{2}{*}{ Age (years) } & Mean $\pm S D$ & $46.3 \pm 6.3$ \\
\hline & Range & $25-56$ \\
\hline \multirow[t]{2}{*}{ Weight (kg) } & Mean $\pm S D$ & $84.49 \pm 7.83$ \\
\hline & Range & $72-111$ \\
\hline \multirow[t]{2}{*}{ Height (cm) } & Mean $\pm S D$ & $168.12 \pm 3.88$ \\
\hline & Range & $160-178$ \\
\hline \multirow[t]{2}{*}{ WC (cm) } & Mean $\pm S D$ & $95.19 \pm 8.27$ \\
\hline & Range & $82-115$ \\
\hline \multirow[t]{2}{*}{ BMI $\left(\mathrm{kg} / \mathrm{m}^{2}\right)$} & Mean \pm SD & $29.74 \pm 2.48$ \\
\hline & Range & $26-38.4$ \\
\hline \multirow[t]{2}{*}{ Obesity } & Overweight & 28 (56.0\%) \\
\hline & Obese & $22(44.0 \%)$ \\
\hline \multirow[t]{2}{*}{ SBP (mmHg) } & Mean \pm SD & $136.8 \pm 16.34$ \\
\hline & Range & $100-170$ \\
\hline \multirow[t]{2}{*}{$\mathrm{DBP}(\mathrm{mmHg})$} & Mean $\pm S D$ & $83.4 \pm 10.32$ \\
\hline & Range & $60-100$ \\
\hline \multirow[t]{2}{*}{ HTN } & No & $32(64.0 \%)$ \\
\hline & Yes & $18(36.0 \%)$ \\
\hline \multirow[t]{2}{*}{$\mathrm{HbA1C}(\%)$} & Mean $\pm S D$ & $6.9 \pm 2.08$ \\
\hline & Range & $4-12$ \\
\hline \multirow[t]{2}{*}{ Diabetes } & Non-diabetic & $21(42.0 \%)$ \\
\hline & Diabetic & $29(58.00 \%)$ \\
\hline \multirow[t]{2}{*}{ FBS (mg/dl) } & Mean +SD & $159.08+61.98$ \\
\hline & Range & $80-261$ \\
\hline \multirow[t]{2}{*}{ 2hPP (mg/dl) } & Mean + SD & $228.5+95.83$ \\
\hline & Range & 100-392 \\
\hline \multirow[t]{2}{*}{$\mathrm{HB}(\mathrm{g} / \mathrm{dl})$} & Mean \pm SD & $11.6 \pm 1.61$ \\
\hline & Range & $5.1-14.01$ \\
\hline \multirow[t]{2}{*}{ TLC (× 109/I) } & Mean $\pm S D$ & $7.73 \pm 1.87$ \\
\hline & Range & $4.2-11.6$ \\
\hline \multirow[t]{2}{*}{ PLT $(\times 109 / I)$} & Mean $\pm S D$ & $228.84 \pm 33.64$ \\
\hline & Range & $169-290$ \\
\hline \multirow[t]{2}{*}{ AST (IU/I) } & Mean $\pm S D$ & $25.9 \pm 5.53$ \\
\hline & Range & $19-38$ \\
\hline \multirow[t]{2}{*}{ ALT (IU/I) } & Mean $\pm S D$ & $29.08 \pm 8.2$ \\
\hline & Range & $13-49$ \\
\hline \multirow[t]{2}{*}{ T. BIL (mg/dl) } & Mean $\pm S D$ & $1.03 \pm 0.09$ \\
\hline & Range & $0.8-1.27$ \\
\hline \multirow[t]{2}{*}{ D. BIL (mg/dl) } & Mean \pm SD & $0.13 \pm 0.09$ \\
\hline & Range & $0.01-0.3$ \\
\hline \multirow[t]{2}{*}{ Albumin (g/l) } & Mean \pm SD & $4.37 \pm 0.31$ \\
\hline & Range & $3.8-4.9$ \\
\hline \multirow[t]{2}{*}{ INR } & Mean \pm SD & $1.04 \pm 0.07$ \\
\hline & Range & $0.9-1.2$ \\
\hline Total cholesterol (mg/dl) & Mean \pm SD & $226.86 \pm 33.72$ \\
\hline & Range & $170-295$ \\
\hline
\end{tabular}

Table 2 (continued)

\begin{tabular}{lll}
\hline & & Total $\mathbf{n o .}=\mathbf{5 0}$ \\
\hline $\mathrm{TG}(\mathrm{mg} / \mathrm{dl})$ & Mean $\pm \mathrm{SD}$ & $202.94 \pm 63.91$ \\
& Range & $120-342$ \\
$\mathrm{LDL}(\mathrm{mg} / \mathrm{dl})$ & Mean $\pm \mathrm{SD}$ & $137.56 \pm 33.42$ \\
& Range & $45-190$ \\
$\mathrm{HDL}(\mathrm{mg} / \mathrm{dl})$ & Mean $\pm \mathrm{SD}$ & $47.89 \pm 15.62$ \\
& Range & $19-86$ \\
\hline
\end{tabular}

Table 3 Adiponectin level and degree of fibrosis according to FibroScan in all studies cases

\begin{tabular}{lll}
\hline & & Total no. $=\mathbf{5 0}$ \\
\hline Adiponectin $(\boldsymbol{\mu g} / \mathbf{m l})$ & Mean \pm SD & \\
& Range & $\mathbf{4 . 0 8} \pm \mathbf{1 . 3 2}$ \\
\hline FibroScan & F0-1 & $31(62.0 \%)$ \\
& F2 & $9(18.0 \%)$ \\
& F3 & $6(12.0 \%)$ \\
FibroScan & F4 & $4(8.0 \%)$ \\
& Low grade & $40(80.0 \%)$ \\
& High grade & $10(20.0 \%)$ \\
\hline
\end{tabular}

high-grade fibrosis patients had adiponectin level equal to or below this value, while it was higher in all our lowgrade fibrosis patients with a sensitivity and specificity of 100\% (Table 11, Fig. 1).

\section{Discussion}

In 2018 , it is estimated that about $25 \%$ of the world population has NAFLD. Several studies have investigated the progression of NAFLD and its subtypes. Although most studies have concluded that non-alcoholic steatohepatitis (NASH) is the progressive form of NAFLD, recent data indicates that a small subset of NAFLD, those with no histologic features of NASH, can progress into advanced liver disease [31].

Adiponectin is the most abundant peptide secreted by adipocytes as well as other cell types including skeletal, cardiac, and endothelial cells. It can protect the liver from injury by enhancing fatty acid $\beta$-oxidation, thus reducing hepatic triacylglycerol content and hepatic insulin resistance. Adiponectin has an anti-inflammatory role by inhibiting the production of pro-inflammatory cytokines such as interleukin-6 (IL-6) and tumor necrosis factor-a (TNF-a) in macrophages and/or reduces their phagocytic capacity. This may be a paramount feature in reversing metabolic dysfunction. Adiponectin potentially has a protective role in insulin resistance (IR) and pathophysiologic procedure of type 2 diabetes mellitus 
Table 4 Comparison between overweight and obese regarding diabetic status and HTN

\begin{tabular}{|c|c|c|c|c|c|c|}
\hline & & $\begin{array}{l}\text { Overweight } \\
\text { No. }=\mathbf{2 8}\end{array}$ & $\begin{array}{l}\text { Obese } \\
\text { No. }=22\end{array}$ & Test value & $P$-value & Sig \\
\hline \multirow[t]{2}{*}{ BMI $\left(\mathrm{kg} / \mathrm{m}^{2}\right)$} & Mean $\pm S D$ & $27.95 \pm 1.01$ & $32.01 \pm 1.85$ & $-9.920^{b}$ & 0.000 & $\mathrm{HS}$ \\
\hline & Range & $26-30$ & $30.1-38.4$ & & & \\
\hline \multirow[t]{2}{*}{ Diabetes } & Non-diabetic & $16(57.1 \%)$ & $5(22.7 \%)$ & $5.990^{\mathrm{a}}$ & 0.014 & $\mathrm{~S}$ \\
\hline & Diabetic & $12(42.9 \%)$ & $17(77.3 \%)$ & & & \\
\hline \multirow[t]{2}{*}{ Hypertension (HTN) } & No & $20(71.4 \%)$ & $12(54.5 \%)$ & $1.524^{\mathrm{a}}$ & 0.217 & NS \\
\hline & Yes & $8(28.6 \%)$ & $10(45.5 \%)$ & & & \\
\hline
\end{tabular}

$P$-value $>0.05$, non-significant; $P$-value $<0.05$, significant; $P$-value $<0.01$, highly significant

${ }^{a}$ Chi-square test

${ }^{\mathrm{b}}$ Independent $t$-test

Table 5 Comparison between overweight and obese cases regarding the degree of liver fibrosis by FibroScan

\begin{tabular}{llllll}
\hline & & $\begin{array}{l}\text { Overweight } \\
\text { No. }=\mathbf{2 8}\end{array}$ & $\begin{array}{l}\text { Obese } \\
\text { No. }=\mathbf{2 2}\end{array}$ & Test value & P-value \\
\hline FibroScan & F0-1 & $22(78.6 \%)$ & $9(40.9 \%)$ & $16.614^{\mathrm{a}}$ & 0.002 \\
& F2 & $4(14.3 \%)$ & $5(22.7 \%)$ & & \\
F3 & $2(7.1 \%)$ & $4(18.2 \%)$ & & \\
& F4 & $0(0.0 \%)$ & $4(18.2 \%)$ & & \\
\hline
\end{tabular}

FibroScan grading for our participants, the exact distribution of grades F0-F4 among both groups

$P$-value $>0.05$, non-significant; $P$-value $<0.05$, significant; $P$-value $<0.01$, highly significant

${ }^{\text {a }}$ Chi-square test

Table 6 Comparison between diabetic and non-diabetic patients regarding obesity and BMI

\begin{tabular}{lllllr}
\hline & & $\begin{array}{l}\text { Non-diabetic } \\
\text { No. }=\mathbf{2 1}\end{array}$ & $\begin{array}{l}\text { Diabetic } \\
\text { No. }=\mathbf{2 9}\end{array}$ & Test value & P-value \\
\hline BMI $\left(\mathrm{kg} / \mathrm{m}^{2}\right)$ & Mean \pm SD & $28.6 \pm 1.81$ & $30.56 \pm 2.6$ & $-2.976^{\mathrm{b}}$ & 0.005 \\
& Range & $26.1-33.1$ & $26-38.4$ & $\mathrm{HS}$ \\
Obesity & Overweight & $16(76.2 \%)$ & $12(41.4 \%)$ & $5.990^{\mathrm{a}}$ & $\mathrm{S}$ \\
& Obese & $5(23.8 \%)$ & $17(58.6 \%)$ & & $\mathrm{S}$ \\
\hline
\end{tabular}

$P$-value $>0.05$, non-significant; $P$-value $<0.05$, significant; $P$-value $<0.01$, highly significant

${ }^{\text {a }}$ Chi-square test

${ }^{\mathrm{b}}$ Independent $t$-test

Table 7 Comparison between diabetic and non-diabetic patients regarding the degree of liver fibrosis by FibroScan

\begin{tabular}{llllll}
\hline & & $\begin{array}{l}\text { Non-diabetic } \\
\text { No. }=\mathbf{2 1}\end{array}$ & $\begin{array}{l}\text { Diabetic } \\
\text { No. }=\mathbf{2 9}\end{array}$ & Test value & P-value \\
\hline FibroScan & F0-1 & $19(90.5 \%)$ & $12(41.4 \%)$ & $22.747^{\text {a }}$ & 0.000 \\
& F2 & $2(9.5 \%)$ & $7(24.1 \%)$ & & \\
F3 & $0(0.0 \%)$ & $6(20.7 \%)$ & & \\
F4 & $0(0.0 \%)$ & $4(13.8 \%)$ & & \\
\hline
\end{tabular}

$P$-value $>0.05$, non-significant; $P$-value $<0.05$, significant; $P$-value $<0.01$, highly significant

${ }^{\text {a }}$ Chi-square test 
Table 8 Comparison between low-grade and high-grade fibrosis regarding demographic, clinical, and laboratory data

\begin{tabular}{|c|c|c|c|c|c|c|}
\hline & & $\begin{array}{l}\text { Low grade } \\
\text { No. }=40\end{array}$ & $\begin{array}{l}\text { High grade } \\
\text { No. }=10\end{array}$ & Test value & $P$-value & $\mathrm{Sig}$ \\
\hline \multirow[t]{2}{*}{ BMI $\left(\mathrm{kg} / \mathrm{m}^{2}\right)$} & Mean $\pm S D$ & $29.11 \pm 1.87$ & $32.27 \pm 3.07$ & -4.176 & 0.000 & HS \\
\hline & Range & $26-33.1$ & $27.2-38.4$ & & & \\
\hline \multirow[t]{2}{*}{ Obesity } & Overweight & $26(65.0 \%)$ & $2(20.0 \%)$ & 6.575 & 0.010 & S \\
\hline & Obese & $14(35.0 \%)$ & $8(80.0 \%)$ & & & \\
\hline \multirow[t]{2}{*}{ Diabetes } & Non-diabetic & $21(52.5 \%)$ & $0(0.0 \%)$ & $9.052^{\mathrm{a}}$ & 0.003 & HS \\
\hline & Diabetic & 19 (47.5\%) & $10(100.0 \%)$ & & & \\
\hline \multirow[t]{2}{*}{ HTN } & No & 29 (72.5\%) & $3(30.0 \%)$ & 6.272 & 0.012 & $S$ \\
\hline & Yes & $11(27.5 \%)$ & $7(70.0 \%)$ & & & \\
\hline
\end{tabular}

$P$-value $>0.05$, non-significant; $P$-value $<0.05$, significant; $P$-value $<0.01$, highly significant

${ }^{a}$ Chi-square test

Table 9 Correlation between adiponectin and clinical features and laboratory investigations of the studied group

\begin{tabular}{|c|c|c|}
\hline & \multicolumn{2}{|c|}{ Adiponectin } \\
\hline & $R$ & $P$-value \\
\hline BMI $\left(\mathrm{kg} / \mathrm{m}^{2}\right)$ & $-0.579^{* *}$ & 0.000 \\
\hline $\mathrm{SBP}(\mathrm{mmHg})$ & $-0.411^{* *}$ & 0.003 \\
\hline $\mathrm{DBP}(\mathrm{mmHg})$ & $-0.322^{*}$ & 0.023 \\
\hline $\mathrm{HbA1C}(\%)$ & $-0.768^{* *}$ & 0.000 \\
\hline Platelets (× 109/l) & $0.833^{* *}$ & 0.000 \\
\hline Albumin $(\mathrm{g} / \mathrm{l})$ & $0.556^{* *}$ & 0.000 \\
\hline Total cholesterol (mg/dl) & $-0.625^{* *}$ & 0.000 \\
\hline $\mathrm{TG}(\mathrm{mg} / \mathrm{dl})$ & $-0.645^{* *}$ & 0.000 \\
\hline LDL (mg/dl) & $-0.653^{* *}$ & 0.000 \\
\hline $\mathrm{HDL}(\mathrm{mg} / \mathrm{dl})$ & $0.601^{* *}$ & 0.000 \\
\hline
\end{tabular}

$P$-value $>0.05$, non-significant; $P$-value $<0.05$, significant; $P$-value $<0.01$, highly significant

Spearman correlation coefficient
(T2DM). Reduction in adiponectin level plays a central role in obesity-related diseases, including IR/T2DM and cardiovascular disease [33].

In this study, a highly significant correlation was found between adiponectin level and liver fibrosis. We found that adiponectin levels were significantly lower in the highgrade fibrosis group with a $P$-value of 0.000 . Similar findings were reported by Nazal et al. [25] who concluded that low adiponectin levels are associated with more severe liver fibrosis in a study that was conducted on seventy (70) obese patients and sixty-nine (69) controls. This can be attributed to the hepatoprotective and antifibrogenic effects of adiponectin in liver injury. It has anti-inflammatory action by neutralizing TNF- $\alpha$ and antifibrotic action by inhibition of HSC proliferation and migration.

On the contrary, Lucero et al. [22] concluded that adiponectin levels were lower in NAFLD patients, but there was no significant relation between adiponectin and the degree of liver fibrosis. That study was conducted on sixty patients with metabolic syndrome of which thirtysix (36) were biopsy-proven NAFLD and twenty-four (24) with no even ultrasound NAFLD evidence. This different result may be due to the difference in the type of patients included in the study.

Table 10 Comparison between adiponectin levels regarding FibroScan results

\begin{tabular}{|c|c|c|c|c|c|c|}
\hline & & \multicolumn{2}{|c|}{ Adiponectin ( $\mu \mathrm{g} / \mathrm{ml})$} & \multirow[t]{2}{*}{ Test value } & \multirow[t]{2}{*}{$P$-value } & \multirow[t]{2}{*}{ Sig } \\
\hline & & Mean \pm SD & Range & & & \\
\hline \multirow[t]{4}{*}{ FibroScan } & F0-1 & $5.4 \pm 0.5$ & $4.3-6$ & 146.027.. & 0.000 & HS \\
\hline & F2 & $3.7 \pm 0.2$ & $3.4-4$ & & & \\
\hline & F3 & $2.1 \pm 0.2$ & $1.9-2.3$ & & & \\
\hline & F4 & $1.6 \pm 0.2$ & $1.4-1.8$ & & & \\
\hline
\end{tabular}

$P$-value $>0.05$, non-significant; $P$-value $<0.05$, significant; $P$-value $<0.01$, highly significant

-Independent $t$-test

..One-way ANOVA test 
Table 11 ROC curve for adiponectin level as a predictor for liver fibrosis

\begin{tabular}{llllll}
\hline Cut off point & AUC & Sensitivity & Specificity & PPV & NPV \\
\hline$\leq 2.31$ & 1.000 & 100.00 & 100.00 & 100.0 & 100.0 \\
\hline
\end{tabular}

In addition, adiponectin level showed a highly significant correlation with obesity. Its mean values in both overweight and obese patients were $4.68 \pm 1.09$ and $3.33 \pm 1.21$, respectively, with a $P$-value of 0.000 . This was consistent with Zhang et al. [34] who found a significant negative correlation between adiponectin and obesity $(P<0.01)$ in a prospective study that was done on a large scale.

This can be attributed to the disproportional accumulation of white adipose tissue in overweight and obese patients which is accompanied by a generalized change in the circulating levels of adiponectin [6].

This study showed that HTN has a significant negative correlation with adiponectin levels and a significant positive correlation with the grade of liver fibrosis $(P=0.01)$. As discussed before, patients with high-grade liver fibrosis have a state of hypo-adiponectinemia, which means loss of the protective role of adiponectin on vascular function.

Highly significant negative correlation was found between SBP and adiponectin levels with a $P$-value of 0.003 . Significant negative correlation was also found between DBP and adiponectin levels with a $P$-value of 0.023. This agreed with Brzeska et al. [4] who found that plasma levels of adiponectin were significantly lower in the patients with hypertension $(P=0.0026)$.
Also, Baden et al. [1] has found that adiponectin showed a significant negative correlation with systolic and diastolic blood pressure $(P<0.01)$. This can be explained by the fact that adiponectin attenuates the phenotype of M1 macrophage which displays upregulation of pro-inflammatory cytokines including TNF- $\alpha$, IL-6, and monocyte chemotactic protein-1 (MCP-1), and it promotes the phenotype of M2 macrophage which upregulates arginase-1 (ARG-1) and interleukin-10 (IL-10). In addition, adiponectin improves the endothelial cell function via increasing nitric oxide (NO) and prostaglandin I2 (PGI2) production, thus exerts a protective action on vascular function through its ability to improve the function of macrophage and endothelial cells [28].

In the present study, a highly significant correlation was found between adiponectin levels and DM with $P$-values of 0.000 being lower in diabetic compared to non-diabetic patients and a highly significant positive correlation between diabetes and high-grade fibrosis with a $P$-value of 0.000 .

This was in agreement with Davis et al. [10], who stated that adiponectin level was negatively correlated with T2DM $(P=0.04)$ and Jaafar et al. [15] who found that diabetic patients presented with more severe fibrosis than non-diabetic NAFLD patients $(P<0.001)$.

T2DM patients can present with high-grade liver fibrosis that could be attributed to glucose intolerance, insulin resistance, and increased serum free fatty acids; there is ectopic fat deposition in visceral organs, notably the liver. Subsequently, fatty liver status increases the liver vulnerability to oxidative stress and mutations, and hence increased inflammation, leading to liver fibrosis [24].

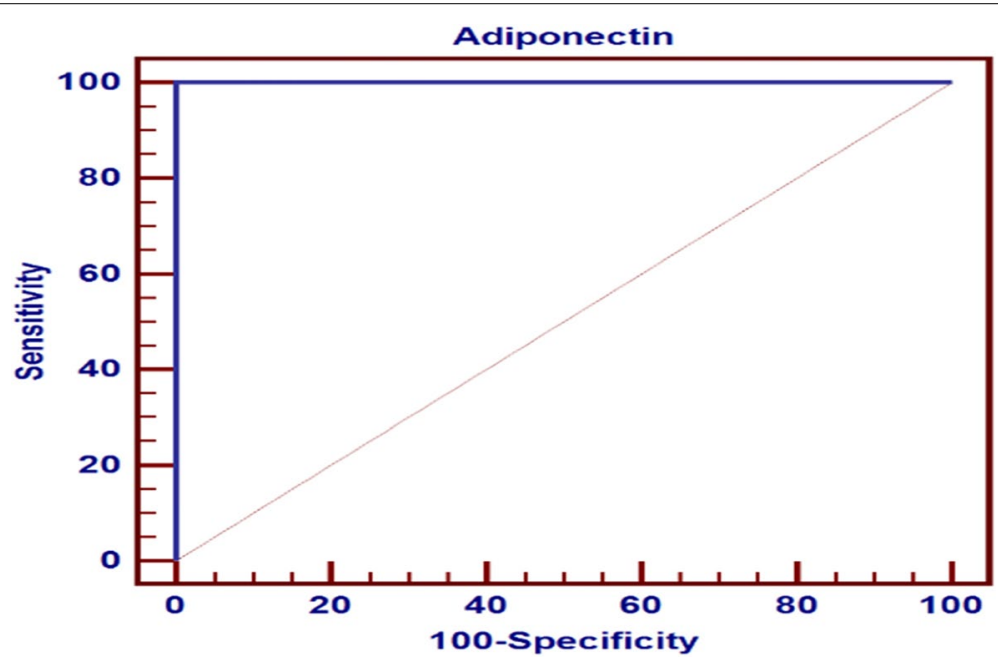

Fig. 1 ROC for adiponectin level as a predictor for liver fibrosis 
This can be attributed to the role of adiponectin in regulating the metabolism of both glucose and lipid in the liver and has been implicated in inhibiting gluconeogenesis, as well as activating fatty acid oxidation and glycolytic pathways which overall improves insulin sensitivity [30].

As for lipid profile, we found that there was a highly significant negative correlation between adiponectin concentration and total cholesterol as well as TG $(P<0.001)$, while there was a highly significant positive correlation between adiponectin and HDL levels $(P<0.001)$. We found that total cholesterol, LDL, and triglycerides were higher among the high-grade fibrosis group.

This was found to be consistent with Brzeska et al. [4], who reported a significant negative correlation between TG and adiponectin level $(P=0.025)$, while a highly significant positive correlation between adiponectin and HDL levels $(P=0.0001)$, but they reported no correlation between adiponectin and total cholesterol levels. Also, Maghsoudi et al. [23] found a significant negative association between adiponectin and TG as well as LDL levels $(P<0.05)$ and a significant positive correlation with HDL $(P<0.05)$.

This agreed with Kumar et al. [20] who found that high stages of liver fibrosis correlated with a significant increase in levels of serum total cholesterol $(P$-value 0.005$)$, TG ( $P$-value 0.002$)$, and LDL ( $P$-value 0.001 ), while an associated significant decrease in HDL $(P$-value 0.001$)$ in NAFLD patients. As explained before, patients with high-grade liver fibrosis have a state of hypoadiponectinemia which has an important role in dyslipidemia.

This is explained by the role of adiponectin in lipid metabolism. It reduces the secretion of hepatic apolipoprotein $\mathrm{E}$ and apolipoprotein $\mathrm{B}$ from the liver. Also, it increases insulin activity, improves glucose tolerance, and plays an important role in fatty acid oxidation; thus, it prevents dyslipidemia [14].

In the current study, more patients with low-grade liver fibrosis were found among the overweight group compared to the obese group (92.95\% compared to 63.6\%), while more high-grade fibrosis was found among the obese group compared to the overweight group (36.4\% compared to $7.1 \%)$. Hence, high-grade fibrosis was positively correlated with obesity in a statistically significant correlation with a $P$-value of 0.01 . This was consistent with Caballería et al. [7] who reported that liver fibrosis in NAFLD patients is correlated with obesity and Ratziu et al. [26] who found that liver fibrosis is significantly correlated with BMI $(P=0.01)$.

This can be explained by the increased visceral fat in obese patients that has a primary role in the pathogenesis of NAFLD. Liver fat accumulation (steatosis) is largely dependent on recirculated free fatty acids (FFA) from the adipose tissue pool. Steatosis leads to lipotoxicity which causes apoptosis, necrosis, generation of oxidative stress, and inflammation. The resulting chronic injury activates a fibrogenic response [11].

In addition, worth noting is another highly significant correlation found between relatively low platelet count and higher grades of liver fibrosis where platelet count were $191.7 \pm 15.02$ in this group compared to $238.31 \pm 30.45$ in the lower grade group with a $P$-value of 0.000 . This agrees with Cao et al. [8] who found that platelet count negatively correlated with the stage of fibrosis in NAFLD patients.

The peripheral platelet production is mainly regulated by thrombopoietin, which is a glycoprotein hormone predominantly synthesized in the liver. During the development and progression of NAFLD, excessive lipid deposition and oxidative stress could impair the mitochondrial functions through inflammatory mediators and then affected thrombopoietin synthesis. Reduced platelet counts could happen in this process [21].

Another similar significant correlation with a $P$-value of 0.001 was established between relatively lower albumin levels and high-grade fibrosis $(4.1 \pm 0.21)$ compared to low-grade fibrosis $(4.44 \pm 0.29)$. This was consistent with Kumar et al. [20] who stated that liver fibrosis is inversely related to serum albumin level in NAFLD patients $(P=0.004)$.

We found that obesity was common among diabetic compared to non-diabetic patients $(58.6 \%$ compared to $23.8 \%)$ as expected with a $P$-value of 0.014 indicating a significant correlation. Consequently, higher BMI values were reported among diabetic patients compared to the non-diabetic group (30.56 \pm 2.6 compared to $28.6 \pm 1.81$, respectively) with a highly significant correlation as indicated by a $P$-value of about 0.005 . This was consistent with Bae et al. [2] who found that a higher BMI than $23.7 \mathrm{~kg} / \mathrm{m}^{2}$ is associated with increased risk of incident diabetes $(P=0.02)$ in a prospective study over 8 years conducted on eight-thousand nine hundred patients. Also, this was in agreement with Riaz et al. [27] who found that BMI in diabetic patients was significantly higher than controls $(P<0.05)$.

In the current study, we found that there is a significant correlation between obesity and high total cholesterol and LDL levels with a $P$-value of 0.031 and 0.023 , respectively, as well as with low HDL levels with a $P$-value of 0.04. These findings were consistent with Maghsoudi et al. [23] who found a significant positive correlation between BMI and total cholesterol and LDL $(P<0.05)$ and a significant negative correlation with HDL $(P<0.05)$. Also, this was in agreement with Hertelyova et al. [13] who found that there was a significant positive correlation between BMI and total cholesterol as well as LDL 
levels $(P<0.001)$, while there was a significant negative correlation between HDL levels and BMI $(P<0.001)$ in a study conducted on four-hundred nineteen patients.

This can be explained by obesity-related uncontrolled increased FFA release from adipose tissue via lipolysis that results in enhanced delivery of FFA to the liver. This leads to increased TG and very low-density lipoprotein (VLDL) production in the liver and inhibition of lipoprotein lipase in adipose tissue and skeletal muscle leading to hypertriglyceridemia. Moreover, the increased VLDL in the liver can inhibit lipolysis of chylomicrons, which also contributes to hypertriglyceridemia. The TG in VLDL is exchanged for cholesteryl esters from LDL and HDL by the cholesteryl ester transport protein, producing TG-rich LDL and HDL. The TG in the LDL and HDL is then hydrolyzed by hepatic lipase, producing both small, dense LDL and HDL [17].

We also found a highly significant correlation between diabetes and total cholesterol, LDL, and TG values which were significantly higher in diabetic compared to the non-diabetic group with $P$-values of 0.000 for all. Higher HDL levels were also reported among non-diabetic compared to the diabetic group ( $55.1 \pm 14.58$ compared to $42.67 \pm 14.42)$ with a $P$-value of 0.004 indicating a highly significant correlation. Kolhar and Priyanka [18] found that dyslipidemia is highly prevalent in diabetics and in particular more prevalent in those with poorly controlled diabetes. Also, Bhowmik et al. [3] reported that diabetic patients had a significant correlation with high total cholesterol, LDL, and TG levels with $P$-values lower than 0.001 for each, while low HDL levels were significantly correlated with diabetes $(P=0.048)$.

The origins of dyslipidemia in diabetes are complex but derived from specific abnormalities in lipoprotein metabolism and abnormalities in insulin action.

In this study, we found that there were low platelet counts in the obese group compared to the overweight group with a $P$-value of 0.003 indicating a highly significant correlation. This did not come in line with Jamshidi and Seif [16] who found that there is a significant relationship between central and general adiposity and higher platelet count. That study was performed on a greater number of cases (four-hundred eighty-six patients) while our study was conducted on fifty patients only. Also, in that study, they excluded diabetic and hypertensive patients. In our study, $77.3 \%$ of our obese patients were diabetics, and $45.5 \%$ of them were hypertensive, while Coban et al. [9] found that there is no significant correlation between platelet count and obesity in a study that was conducted on one-hundred obese patient and one-hundred controls.

Another noted highly significant correlation was found between lower platelet count and diabetes with a $P$-value of about 0.000 . This may be because of various factors such as high production and turnover rate in T2DM with diminished mean platelet survival [19].

This goes with Kshirsagar et al. [19] who reported decreased platelet count in diabetic compared to nondiabetic patients but was not statistically significant $(P=0.08)$, as well as with Buch et al. [5] and Hekimsoy et al. [12].

We also found relatively lower serum albumin levels in the diabetic compared to the non-diabetic group with a $P$-value of 0.000 . This agrees with Riaz et al. [27] who found that serum albumin for diabetic individuals was lower than control subjects which was statistically significant correlation $(P<0.05)$. Mostly due to the fact that patients with T2DM with long duration and/or poor control may develop diabetic nephropathy where they lose albumin in urine.

\section{Conclusion}

Lower serum adiponectin level is a predictor of NAFLD. Low serum adiponectin level should be considered as a potential non-classical biomarker of metabolic complications in patients with NAFLD, although liver biopsy still remains the gold standard for the diagnosis of NAFLD and the staging of fibrosis and FibroScan appears to be an acceptable non-invasive tool for the assessment of fibrosis in NAFLD patients. We found a highly significant negative correlation between adiponectin and DM, hypertension, and obesity. Yet, serum adiponectin measurement could be of value in detecting patients with more advanced fibrosis among all patients with NAFLD. It is especially useful in excluding patients with advanced fibrosis. Its diagnostic accuracy is excellent and is better than other noninvasive predictors.

\footnotetext{
Abbreviations

NAFLD: Non-alcoholic liver disease; AST: Aspartate aminotransferase; ALT: Alanine aminotransferase; BMI: Body mass index; NFa: Nuclear factor $\alpha$; TGF $\beta$ Transforming growth factor $\beta$; PAI-1: Plasminogen activator inhibitor-1; HSCs: Hepatic stellate cells; AMPK: Adenosine monophosphate-activated protein kinase; $a-S M A$ : Alpha-smooth muscle actin; SBP: Systolic blood pressure; DBP: Diastolic blood pressure; CBC: Complete blood count; HBsAg: Hepatitis B surface antigen; HCV-Ab: Hepatitis C virus antibody; HbA1C: Glycosylated hemoglobin; INR: International normalization ratio; ELISA: Enzyme-linked immunosorbent assay; 2hPP: Two-hour post-prandial; PPV: Positive predictive value; NPV: Negative predictive value; ROC: Receiver operating characteristic; HTN: Hypertension; DM: Diabetes mellitus; HDL: High-density lipoprotein; TG: Triglyceride; LDL: Low-density lipoprotein; NASH: Non-alcoholic steatohepatitis; IL-6: Interleukin-6; TNF-a: Tumor necrosis factor-a; IR: Insulin resistance; T2DM: Type 2 diabetes mellitus; MCP-1: Monocyte chemotactic protein-1; ARG-1: Arginase-1; IL-10: Interleukin-10; NO: Nitric oxide; PGI2: Prostaglandin 12; FFA: Free fatty acids; VLDL: Very low-density lipoprotein.
}

Acknowledgements

None. 


\section{Authors' contributions}

MM formulated the research idea and shared in the data analysis and manuscript revision. TY supervised the data analysis and manuscript drafting. EA shared in the data collection, statistical analysis, and manuscript drafting. AA shared in the formulation of research hypothesis, data processing, statistical analysis, and drafting of the final manuscript. All authors have read and approved the final manuscript.

\section{Funding}

Not applicable.

\section{Availability of data and materials}

The datasets used and analyzed during the current study are available from the corresponding author upon reasonable request.

\section{Declarations}

Ethics approval and consent to participate

All subjects involved in the study signed an informed consent to participate. The study was approved by the Ethical Review Board of Ain Shams University. The study protocol conforms to the ethical guidelines of the Declaration of Helsinki as reflected in a priori approval by the institution's human research committee.

\section{Consent for publication}

Not applicable.

\section{Competing interests}

All authors declare that they have no competing interests.

\section{Author details}

${ }^{1}$ Gastroenterology, Hepatology and Internal Medicine Department, Ain Shams University, Cairo, Egypt. ${ }^{2}$ El Obour City, Egypt.

Received: 5 February 2021 Accepted: 14 July 2021

Published online: 21 September 2021

\section{References}

1. Baden MY, Yamada Y, Takahi Y et al (2013) Association of adiponectin with blood pressure in healthy people. Clin Endocrinol 78(2):226-231

2. Bae JC, Cho NH, Kim JH et al (2020) Association of body mass index with the risk of incident type 2 diabetes, cardiovascular disease, and all-cause mortality: a community-based prospective study. Endocrinol Metab 35(2):416-424

3. Bhowmik B, Siddiquee T, Mujumder A et al (2018) Serum lipid profile and its association with diabetes and prediabetes in a rural Bangladeshi population. Int J Environ Res Public Health 15(9):1944

4. Brzeska A, Sołtysiak M, Ziemak J et al (2018) Plasma adiponectin in hypertensive patients with and without metabolic syndrome. Arterial Hypertension 22(1):29-36

5. Buch A, Kaur S, Nair R et al (2017) Platelet volume indices as predictive biomarkers for diabetic complications in type 2 diabetic patients. J Lab Phys 9(2):84-88

6. Burhans MS, Hagman DK, Kuzma JN et al (2011) Contribution of adipose tissue inflammation to the development of type 2 diabetes mellitus. Compr Physiol 9(1):1-58

7. Caballería L, Pera G, Arteaga I et al (2018) High prevalence of liver fibrosis among European adults with unknown liver disease: a population-based study. Clin Gastroenterol Hepatol 16(7):1138-1145

8. Cao W, Zhao C, Shen C et al (2013) Cytokeratin 18, alanine aminotransferase, platelets and triglycerides predict the presence of nonalcoholic steatohepatitis. PLoS ONE 8(12):e82092

9. Coban E, Ozdogan M, Yazicioglu G et al (2005) The mean platelet volume in patients with obesity. Int J Clin Pract 59:981-982

10. Davis SK, Gebreab SY, Xu R et al (2015) Association of adiponectin with type 2 diabetes and hypertension in African American men and women: the Jackson Heart Study. BMC Cardiovasc Disord 15(1):13

11. Di Ciaula A, Baj J, Garruti G et al (2020) Liver steatosis, gut-liver axis, microbiome and environmental factors. A never-ending bidirectional cross-talk. J Clin Med 9(8):2648
12. Hekimsoy Z, Payzin B, OrnekT et al (2004) Mean platelet volume in type 2 diabetic patients. J Diabetes Complications 18:173-176

13. Hertelyova Z, Salaj R, Chmelarova A et al (2016) The association between lipid parameters and obesity in university students. J Endocrinol Invest 39:769-778

14. Izadi V, Farabad E, Azadbakht L (2013) Epidemiologic evidence on serum adiponectin level and lipid profile. Int J Prev Med 4(2):133

15. Jaafar RF, Ali AM, Zaghal AM et al (2019) Fibroscan and low-density lipoprotein as determinants of severe liver fibrosis in diabetic patients with nonalcoholic fatty liver disease. Eur J Gastroenterol Hepatol 31(12):1540-1544

16. Jamshidi L and Seif A (2017): Association between obesity, white blood cell and platelet count. Zahedan J Res Med Sci. 19: e4955.

17. Jung UJ, Choi MS (2014) Obesity and its metabolic complications: the role of adipokines and the relationship between obesity, inflammation, insulin resistance, dyslipidemia and nonalcoholic fatty liver disease. Int J Mol Sci 15(4):6184-6223

18. Kolhar U, Priyanka P (2017) Study of serum lipid profile in type 2 diabetes mellitus patients and its association with diabetic nephropathy. Int J Adv Med 4(6):1513-1516

19. Kshirsagar RM, Deoke S, Akhtar S (2019) Platelet indices in type 2 diabetes mellitus and their association with microvascular complications. Panacea J Med Sci 9(1):23-28

20. Kumar R, Rastogi A, Sharma MK et al (2013) Liver stiffness measurements in patients with different stages of nonalcoholic fatty liver disease: diagnostic performance and clinicopathological correlation. Dig Dis Sci 58(1):265-274

21. Liu F, Zhou H, Cao L et al (2018) Risk of reduced platelet counts in patients with nonalcoholic fatty liver disease (NAFLD): a prospective cohort study. Lipids Health Dis 17(1):221

22. Lucero D, Miksztowicz V, Gualano G et al (2017) Nonalcoholic fatty liver disease associated with metabolic syndrome: influence of liver fibrosis stages on characteristics of very low-density lipoproteins. Clin Chim Acta 473:1-8

23. Maghsoudi Z, Kelishadi R, Hosseinzadeh-Attar MJ (2016) The comparison of chemerin, adiponectin and lipid profile indices in obese and non-obese adolescents. Diabetes Metab Syndr 10(2):S43-\$46

24. Marchisello S, Di Pino A, Scicali R et al (2019) Pathophysiological, molecular and therapeutic issues of nonalcoholic fatty liver disease: an overview. Int J Mol Sci 20(8):1948

25. Nazal L, Riquelme A, Solís N et al (2010) Hypoadiponectinemia and its association with liver fibrosis in morbidly obese patients. Obes Surg 20(10):1400-1407

26. Ratziu V, Giral P, Charlotte F et al (2000) Liver fibrosis in overweight patients. Gastroenterology 118(6):1117-1123

27. Riaz H, Khan MS, Siddiqi TJ et al (2018) Association between obesity and cardiovascular outcomes: a systematic review and meta-analysis of Mendelian randomization studies. JAMA Netw Open 1(7):e183788

28. Rojas E, Rodríguez-Molina D, Bolli P et al (2014) The role of adiponectin in endothelial dysfunction and hypertension. Curr Hypertens Rep 16(8):463

29. Satapathy SK, Sanyal AJ (2015) Epidemiology and natural history of nonalcoholic fatty liver disease. Semin Liver Dis 35:221-235

30. Stern JH, Rutkowski JM, Scherer PE (2016) Adiponectin, leptin, and fatty acids in the maintenance of metabolic homeostasis through adipose tissue crosstalk. Cell Metab 23(5):770-784

31. Sumida Y, Yoneda M (2018) Current and future pharmacological therapies for NAFLD/NASH. J Gastroenterol 53:362-376

32. Udomsinprasert W, Honsawek S, Poovorawan Y (2018) Adiponectin as a novel biomarker for liver fibrosis. World J Hepatol 10(10):708

33. Yosaee S, Khodadost M, Esteghamati A et al (2019) Adiponectin: an indicator for metabolic syndrome. Iran J Public Health 48(6):1106-1115

34. Zhang H, Niu Y, Gu H et al (2019) Low serum adiponectin is a predictor of progressing to nonalcoholic fatty liver disease. J Clin Lab Anal 33(3):e22709

35. Zhao L, Fu Z, Liu Z (2014) Adiponectin and insulin cross talk: the microvascular connection. Trends Cardiovasc Med 24(8):319-324

\section{Publisher's Note}

Springer Nature remains neutral with regard to jurisdictional claims in published maps and institutional affiliations. 